\title{
Velocity Measurement by PIV Using W-Shaped Scanning Light Sheet*
}

\author{
Shumpei Funatani, Tetsuaki Takeda, Koji Toriyama \\ Department of Mechanical Systems Engineering, University of Yamanashi, Kofu, Japan \\ Email: sfunatani@yamanashi.ac.jp
}

Received 3 August 2014; accepted 1 January 2015; published 26 January 2015

Copyright (C) 2015 by authors and Scientific Research Publishing Inc.

This work is licensed under the Creative Commons Attribution International License (CC BY). http://creativecommons.org/licenses/by/4.0/

c) (i) Open Access

\begin{abstract}
This study proposes a three-dimensional (3D) particle image velocimetry (PIV) method using Wshaped light sheet and color PIV with a digital SLR camera. The uncertainty of the velocity measurement was also studied and it was acceptable. The spatial resolution of the z-direction has much room for improvement by increasing the number of cameras. When applied to the velocity distribution measurement of a thermal vertical buoyant plume, the proposed 3D PIV method is found to be very effective for studying thermal structures and well suited for measuring the airflow velocity field.
\end{abstract}

\section{Keywords}

\section{D PIV, Color PIV, W-Shaped Light Sheet, Airflow}

\section{Introduction}

To understand the transport phenomena of heat and fluid flow in various fields of engineering, it is essential to develop experimental methods for measuring the temperature and velocity field of the fluid flow. For example, by spraying a mist of propylene glycol into the airflow, two-color laser-induced fluorescence (LIF) can be used to measure the temperature of the airflow [1].

The instantaneous measurement of three-dimensional three-component (3D-3C) velocity fields is essential for studying various fluid flows. For this purpose, a tomographic particle image velocimetry (PIV) method was developed, which was successfully applied to an actual flow field [2]. However, this method uses volumetric illumination and requires extremely high power for the velocimetry of airflow. Moreover, several high-resolution cameras are required for obtaining original images of the tomographic PIV, and this method becomes cost prohibitive.

In conventional PIV, the laser light sheet used for visualization is flat and can only be used to visualize a single two-dimensional (2D) plane. The visualized area can be extended to a three-dimensional (3D) field by ap"Short paper.

How to cite this paper: Funatani, S., Takeda, T. and Toriyama, K. (2015) Velocity Measurement by PIV Using W-Shaped Scanning Light Sheet. Journal of Flow Control, Measurement \& Visualization, 3, 35-39. 
plying a pair of scanning light sheets [3]. However, the scanning velocity is limited by the frame rate of the camera. To solve these problems, we propose a new method for instantaneously visualizing the entire 3D field by deforming the light sheet to a W-shape. In this technique, the light sheet is traversed, and the trajectory generates cyclic ramp waves. Using a $\mathrm{W}$-shaped light sheet, we were able to simultaneously visualize different parts of the field in the x-, y-, and z-direction. The velocity distributions could, therefore, be obtained where the $\mathrm{W}$-shaped light sheet intersected the flow field, and the 3D velocity distribution could be evaluated by interpolating the velocity distributions on each $\mathrm{W}$-plane.

However, the proposed PIV method reduces the number of velocity vectors in the $x$-y plane and significantly deforms the shape of the correlation area. We must increase the number of pixels of the camera to maintain the good spatial resolution found in traditional PIV velocity distributions. Recently, the number of pixels of digital single-lens reflex (SLR) cameras has increased, and their emerging large-scale commercial production has been accompanied by reduced cost. The number of pixels in a commercially available digital SLR camera is presently over 24,000,000, and the sensitivity exceeds the requirements of ISO 25,600. If necessary, however, the number of pixels can be easily increased by lining up multiple cameras. A two-color PIV system using an ND:YAG laser [4] or Ar-ion laser [5] has been previously proposed, but the wavelength of the lasers was not optimal for the wavelength range of red-green-blue (RGB) filters in color charge-coupled device (CCD) cameras. Recently, various diode lasers have been developed with wavelengths that cover the visible light range and have power in excess of thousands of milliwatts. These diode lasers can be used to create a color PIV system for measuring the velocity field of airflow by illuminating different colors of diode lasers at defined time intervals.

The steps for reliably applying the proposed 3D PIV are given as follows:

1) Develop a method for generating a W-shaped light sheet of scanning laser beams using a rotating mirror.

2) Establish the color PIV method for the digital SLR camera.

3) Evaluate the uncertainty of this color PIV technique.

In this study, we demonstrate these steps and apply the 3D-2C PIV technique to a vertical buoyant plume.

\section{Measurement Methods}

\subsection{Color PIV Method Using Digital SLR Camera}

In this 3D PIV technique, it is necessary to generate a deformed light sheet and illuminate the visualized area twice within a given time interval. To satisfy these requirements, we developed a color PIV method using two rotating mirrors, a dichroic mirror, and two diode lasers. The experimental setup and visualized image are shown in Figure 1. A dichroic mirror was used to combine two lasers to form a single red and blue laser. The optical axes of each laser were subtly shifted to create a time delay while scanning. A color image was taken with subtle displacement of each color image. The red and blue pixels were arrayed using a Bayer matrix pattern, and the color image was generated by interpolating each combination of pixels. Each color component is divided, and direct cross-correlation method and sub-pixel analysis [3] were applied between each image.

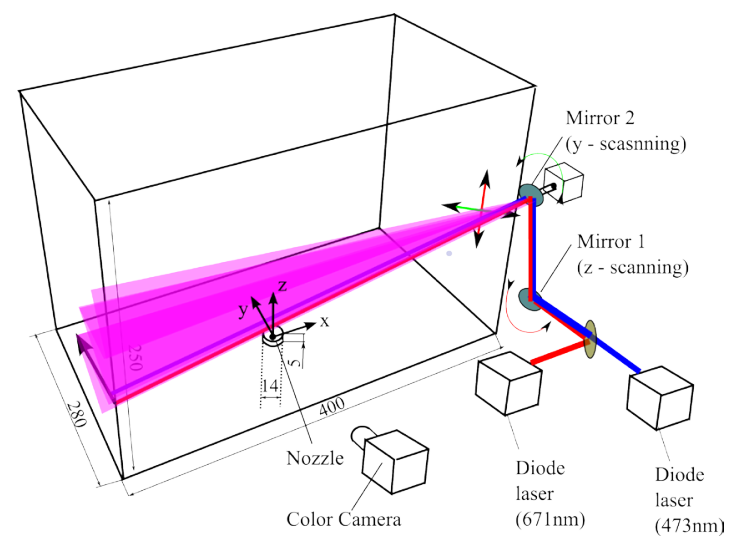

(a)

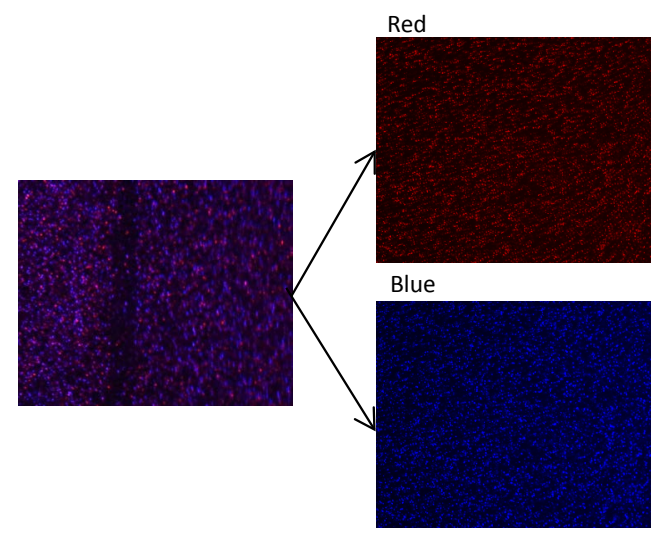

(b)

Figure 1. Experimental apparatus and visualized image for PIV using W-shaped scanning light sheet (unit in mm). (a) Experimental setup; (b) Visualized images. 


\subsection{Experimental Setup of 3D PIV Technique Using W-Shaped Light Sheet}

The experimental apparatus used for the 3D PIV is shown in Figure 1(a). The volume of the test section was $400 \times 250 \times 280 \mathrm{~mm}^{3}$. The test section was visualized using two diode lasers. The two illumination colors, namely red and blue, were generated by diode lasers with wavelengths of 671 and $473 \mathrm{~nm}$, respectively. The visualization images were obtained by a digital SLR camera with color (red, green, and blue) pixel image grids. The illumination power of the red laser was set to $500 \mathrm{~mW}$ and that of blue laser was adjusted to equalize the intensity of color in the visualization images. The laser light sheet was generated by scanning a laser beam using rotating mirrors. A digital SLR camera (Nikon D7100, 14 bit, $6000 \times 4000$ pixels, ISO 25,600) and a long focal length macro lens (Nikon Micro-Nikkor, $f=200 \mathrm{~mm}$ ) were used to record the visualized image. The measurement volume size was $36 \times 20 \times 42 \mathrm{~mm}^{3}$, and four slices of the W-shaped light sheet were recorded in a single image. The measurement volume and the number of slices can be extended using multiple cameras. The optical axis of the camera was set to the positive y-direction. The F-number of the camera was set to 32 to minimize the defocusing due to the deformation of the light sheet. The time chart of the $\mathrm{x}$ - and z-positions of each laser beam controlled by rotating mirrors is shown in Figure 2. The shutter of the camera was kept open during laser illumination using the release of digital SLR camera. Therefore, the accuracy of the shutter timing control is not influenced by the uncertainty of the velocity measurement. The trajectory of each color beam shows that each beam passes through the same position in a given time interval; each visualized image is taken, and the time interval is constant over the whole flow field. The PIV technique was applied between each visualized image, and the velocity distributions are interpolated to generate a 3D velocity field. Calibration of the actual position of each visualized plane and the pixel positions on the visualized image was evaluated by moving a calibration plate (x-z plane) in the y-direction [6]. The flow chart in Figure 4 shows the reconstruction procedure for the velocity distribution in a 3D field [7]. The actual positions of the visualized field are previously known from the rotation angle of each rotating mirror (Figure 3(a)). The visualized image is taken on the flat image plane (Figure 3(b)). Therefore, PIV analysis can be applied to the visualized image (Figure 3(c)). Each velocity vector is allocated on the actual position (Figure 3(d)). In this study, the xz-component (u, w) velocity distribution, which is deformed in a W-shape, is generated. The three components of the velocity distribution can be evaluated using two cameras and by applying stereoscopic PIV, as shown in Figure 3(c).

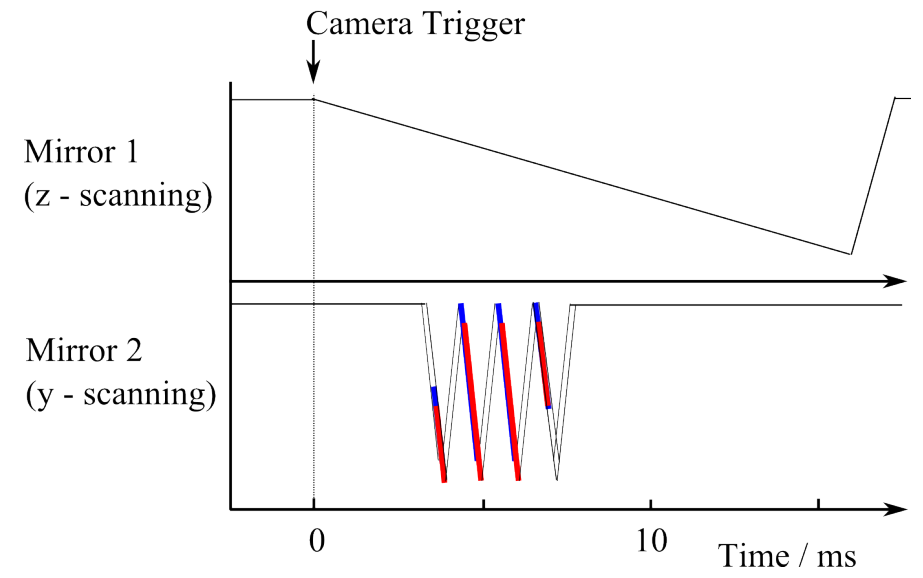

Figure 2. Time chart of the $\mathrm{z}$ - and y-positions of each laser beam.

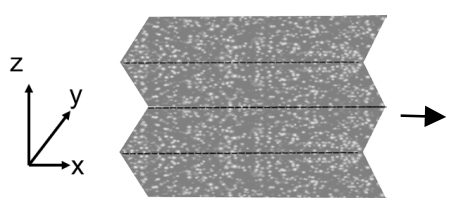

(a)

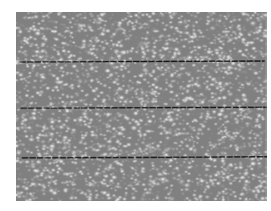

(b)

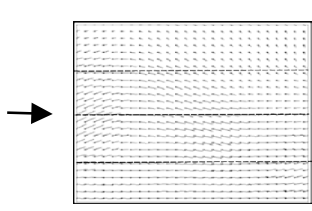

(c)

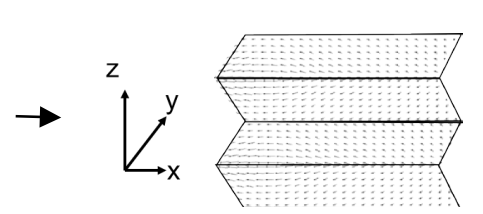

(d)

Figure 3. Flow chart of the 3D reconstruction of velocity field. (a) Visualized sections; (b) Visualized images; (c) Velocity distribution; (d) Reconstructed velocity field (u, w). 


\section{Results}

\subsection{D PIV Method Using W-Shaped Light Sheet and Color PIV Method}

Figure 4 shows the 3D velocity distribution of the $\mathrm{z}$-component $(\mathrm{w})$ velocity distribution in the vertical jet. The temperature of the atmosphere was $\mathrm{T}_{\mathrm{C}}=25^{\circ} \mathrm{C}$, and a buoyant plume was generated from a circular pipe, which has an inside diameter of $\mathrm{D}=14 \mathrm{~mm}$, and was located at the bottom surface of the test section. The temperature of hot air in the nozzle was $\mathrm{T}_{\mathrm{h}}=35^{\circ} \mathrm{C}$ at $\mathrm{y}=-10 \mathrm{~mm}$, and the average velocity was $\mathrm{V}_{0}=10 \mathrm{~mm} / \mathrm{s}$. Therefore, the Froude number of the vertical buoyant plume was $\mathrm{Fr}_{0}=4.23$, and the Reynolds number was $\operatorname{Re}=1670$. The instantaneous structure of the vertical plume generated by the nozzle and its interaction with the stagnant surrounding air as well as the velocity distribution were steady which was the same as previous study [8]. Therefore, these conditions were sufficient for evaluating the 3D PIV measurement method.

\subsection{Uncertainty Analysis of the Color PIV Method}

As part of our assessment of the proposed velocity measurement technique, we investigated the velocity measurement uncertainty. The uncertainty originates from error sources such as the camera calibration, image processing, pulse delay, and response of the tracer [9]. The differences between regular PIV and the beamscanning color PIV are the pulse time and sensitivity coefficient. The uncertainty of the pulse time is larger than that of regular PIV because the pulse timing is decided by the timing of the passing laser beam and its uncertainty influences the diameter and scanning speed of the laser beam. On the contrary, the sensitivity coefficient becomes lower because the number of image pixels is larger using digital SLR camera.

As a result, the total uncertainty interval of velocity was $0.58 \mathrm{~mm} / \mathrm{s}$. In this study, the influence of image distortion became sensitive because the camera had high resolution. Therefore, a long focal length macro lens was used to reduce the influence of image distortion. In this study, four slices of the visualized plane were used, and the interval of slices was $14 \mathrm{~mm}$. The spatial resolution of z-direction can be improved by increasing the number of slices. To do this, it is necessary to increase the magnification of the camera lens and to increase the number of cameras.

\section{Conclusion}

A 3D-2C PIV method, which utilized color PIV with a digital SLR camera and dual-beam scanning, was proposed and its uncertainty was studied. The results show that the total uncertainty is acceptable, but the spatial resolution of the $\mathrm{z}$-direction needs to be improved by increasing the number of cameras. This method could be applied to investigate the heat transfer and fluid flow characteristics of a vertical buoyant plume. The number of cameras can be easily increased because the accuracy of camera timing control of this PIV technique is allowed to be lower, and we can apply stereoscopic PIV techniques using multiple cameras.

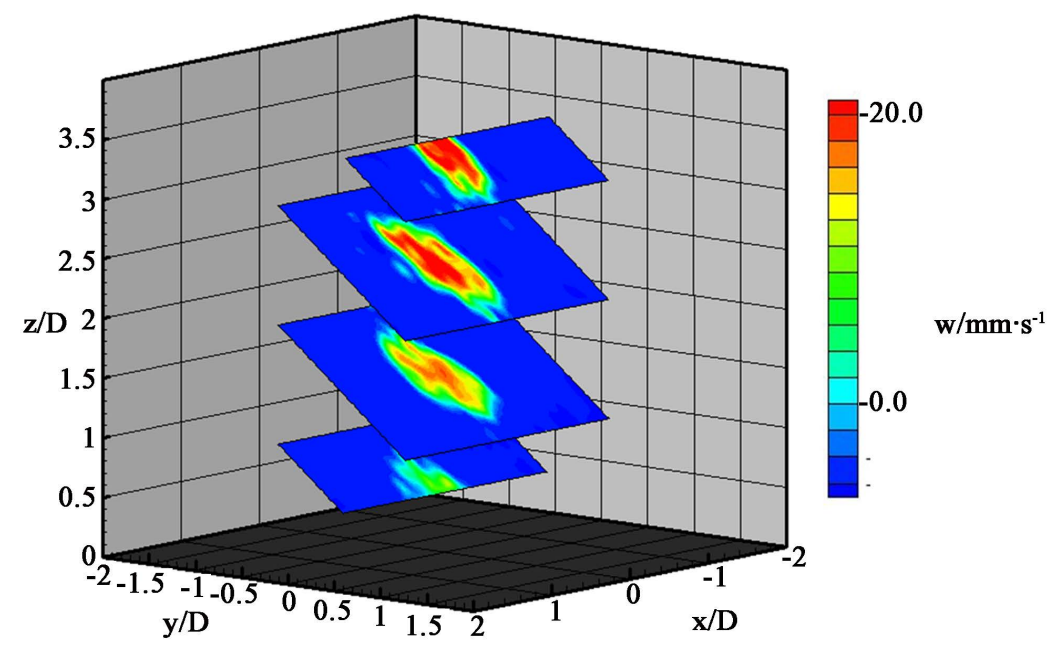

Figure 4. 3D velocity distribution in the vertical jet. 


\section{Acknowledgements}

This work was supported by JSPS KAKENHI Grant Number 24760131.

\section{References}

[1] Funatani, S., Toriyama, K. and Takeda, T. (2013) Temperature Measurement of Air Flow Using Fluorescent Mists Combined with Two-Color LIF. Journal of Flow Control, Measurement \& Visualization, 1, 20. http://dx.doi.org/10.4236/jfcmv.2013.11004

[2] Elsinga, G.E., Scarano, F., Wieneke, B. and Van Oudheusden, B.W. (2006) Tomographic Particle Image Velocimetry. Experiments in Fluids, 41, 933-947. http://dx.doi.org/10.1007/s00348-006-0212-z

[3] Fujisawa, N. and Funatani, S. (2000) Simultaneous Measurement of Temperature and Velocity in a Turbulent Thermal Convection by the Extended Range Scanning Liquid Crystal Visualization Technique. Experiments in Fluids, 29, S158-S165. http://dx.doi.org/10.1007/s003480070017

[4] Gogineni, S., Goss, L., Pestian, D. and Rivir, R. (1998) Two-Color Digital PIV Employing a Single CCD Camera. Experiments in Fluids, 25, 320-328. http://dx.doi.org/10.1007/s003480050236

[5] Post, M.E., Trump, D.D., Goss, L.P. and Hancock, R.D. (1994) Two-Color Particle-Imaging Velocimetry Using a Single Argon-Ion Laser. Experiments in Fluids, 16, 263-272.

[6] Fujisawa, N., Funatani, S. and Katoh, N. (2005) Scanning Liquid-Crystal Thermometry and Stereo Velocimetry for Simultaneous Three-Dimensional Measurement of Temperature and Velocity Field in a Turbulent Rayleigh-Bernard Convection. Experiments in Fluids, 38, 291-303. http://dx.doi.org/10.1007/s00348-004-0891-2

[7] Funatani, S. and Takeda, T. (2013) 3D-3C PIV Method by Using W-Shaped Light Sheet and Color PIV. 10th International Symposium on Particle Image Velocimetry—PIV13, Delft, 2-4 July 2013, Paper No. A094.

[8] Watanabe, R., Gono, T., Yamagata, T. and Fujisawa, N. (2015) Three-Dimensional Flow Structure in Highly Buoyant Jet by Scanning Stereo PIV Combined with POD Analysis. International Journal of Heat and Fluid Flow, 52, 98-110. http://dx.doi.org/10.1016/j.ijheatfluidflow.2014.12.003

[9] Raffel, M., Willert, C. and Kompenhans, J. (1998) Particle Image Velocimetry: A Practical Guide. Springer-Verlag, Berlin. http://dx.doi.org/10.1007/978-3-662-03637-2 
Scientific Research Publishing (SCIRP) is one of the largest Open Access journal publishers. It is currently publishing more than 200 open access, online, peer-reviewed journals covering a wide range of academic disciplines. SCIRP serves the worldwide academic communities and contributes to the progress and application of science with its publication.

Other selected journals from SCIRP are listed as below. Submit your manuscript to us via either submit@scirp.org or Online Submission Portal.
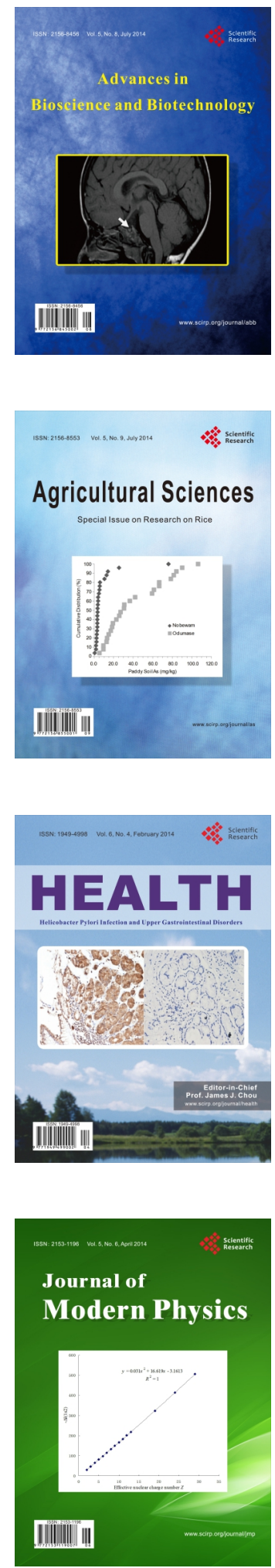
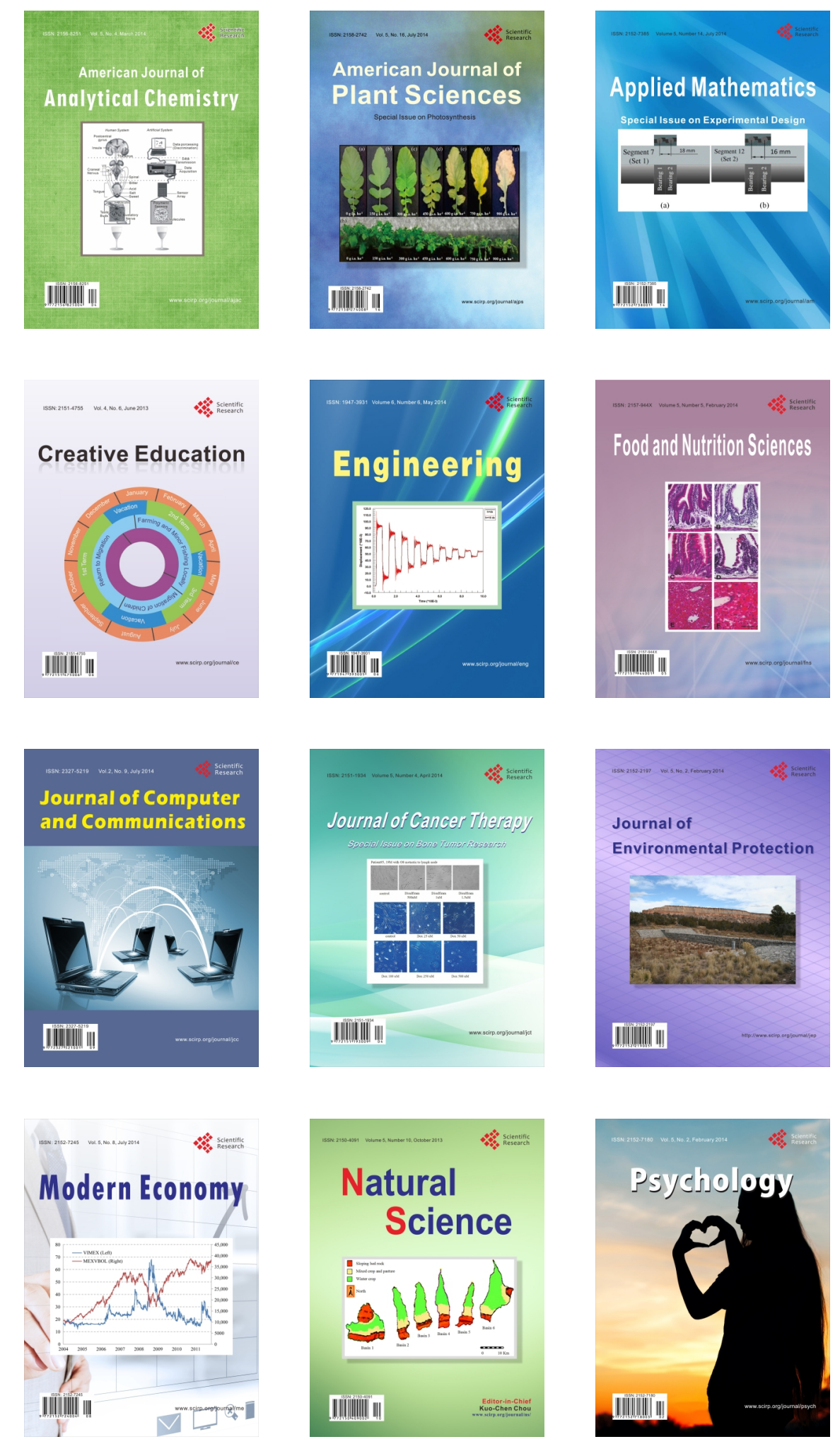